\title{
Consumer perceptions of anticake agents on shredded Cheddar cheese
}

\section{S. E. Meals, W. S. Harwood, and M. A. Drake* (i)}

Department of Food, Bioprocessing, and Nutrition Sciences, Southeast Dairy Foods Research Center, North Carolina State University, Raleigh, 27695

\section{ABSTRACT}

Prepackaged natural cheese shreds are a growing consumer category. Anticake agents are applied to commercial cheese shreds to assist with shelf life and ease of use. The objective of this study was to investigate consumer perception of 3 anticake agents applied at various levels to Cheddar cheese shreds. Three common anticake agents $(80 \%$ potato starch $/ 20 \%$ cellulose blend, $100 \%$ potato starch, or potato starch/corn starch/calcium sulfate blend) were applied to duplicate lots of Cheddar cheese shreds at 1, 2, 3, 4, and 5\% (wt/ wt). Control Cheddar cheese shreds with no anticake were also included. Sensory properties (appearance, flavor, texture, and hot texture) were documented using a trained sensory panel $(\mathrm{n}=8)$, and 3 consumer acceptance tests were also conducted. In test 1, consumers (n $=110$ ) visually evaluated liking of cold shred appearance. In test 2 , consumers $(\mathrm{n}=100)$ evaluated melted shreds on a flour tortilla for overall liking and appearance, flavor, and texture liking. In test 3, consumers $(\mathrm{n}=49)$ participated in a home usage test. Two-way ANOVA (anticake $\times$ anticake application rate) was used to interpret the collected data from each test. Visual appearance of shreds was the primary attribute influenced by anticake application and anticake agent. Trained panel evaluation demonstrated that the $100 \%$ potato starch anticake had minimal effects on visual appearance. The other 2 agents $(80 \%$ potato starch $/ 20 \%$ cellulose blend and potato starch/corn starch/calcium sulfate blend) showed increases in visible powder at $>3 \%$ (wt/wt). Consistent with results from trained panelists, higher application rates decreased consumer appearance and color liking for Cheddar shreds with $80 \%$ potato starch $/ 20 \%$ cellulose and potato starch/ corn starch/calcium sulfate blends at $>2$ or $3 \%$ (wt/ wt), respectively. Appearance liking of melted shreds decreased with increased anticake application percent but decreased the most for $100 \%$ potato starch anti-

Received June 8, 2020.

Accepted July 26, 2020.

*Corresponding author: mdrake@ncsu.edu cake at greater than $1 \%(\mathrm{wt} / \mathrm{wt})$ application. Overall liking, flavor liking, and texture liking attributes for melted shreds were negatively affected at $>3 \%$ (wt/ wt) application regardless of anticake agent used. In general, anticake agents can be applied to Cheddar cheese shreds at up to $3 \%$ (wt/wt) with minimal effect on consumer perception.

Key words: Cheddar cheese shreds, consumer liking, anticake agents

\section{INTRODUCTION}

Cheese sales in the United States increased by $10 \%$ from 2012 to 2017, with total category sales expected to rise an additional $8 \%$, totaling $\$ 25.5$ billion in annual sales by 2022 (Mintel Group Ltd., 2017). According to the USDA, Cheddar cheese consumption was second only to mozzarella, with approximately $4.7 \mathrm{~kg}$ consumed per capita per year versus $5.3 \mathrm{~kg}$ per year, respectively (USDA-ERS, 2018). The growth in cheese consumption in the United States over the past few years is primarily driven by flavor, with roughly $80 \%$ of cheese consumers citing taste as their primary purchase motivation (Mintel Group Ltd., 2017). Consumers crave convenience and are willing to pay for it. For the US cheese market, convenience has become the most influential trend of 2018 (Canning, 2018). This focus on convenience has increased shredded natural cheese sales to the highest selling subcategory of cheese at $\$ 4,735$ million in 2018 (Canning, 2018). However, manufacture of cheese shreds poses additional challenges compared with their block counterparts.

Cheese shreds have additional characteristics that affect consumer liking, including shred length, width, and anticake agents. Anticake agents are ingredients added to cheese shreds to prevent clumping or agglomeration during the shredding process, storage, and shipping. Clumping decreases ease of consumer use and may pose microbial concerns. Anticake agents are typically combinations or sole applications of potato starch, corn starch, calcium sulfate, or powdered cellulose, to prevent shreds from sticking and caking (Elayedath and Barringer, 2002). Additionally, natamycin, a com- 
mon mold inhibitor, is added to help prolong shelf life (Elayedath and Barringer, 2002). Natamycin can be applied to cheese as an antimycotic at a maximum of 20 $\mathrm{mg} / \mathrm{kg}$ of the finished product (21CFR172.115; FDA, 2016a).

Multiple concerns surround the use of anticake agents for cheese shreds. Application of anticake agents adds to production costs, increases dust during manufacture (a possible health hazard to workers), and introduces additional risk of bacterial contamination. However, it should be noted that anticake ingredients may be of lower cost than the cheese itself, and the benefits provided by the anticake ingredients due to increased throughput (less sticking and clogging) provide additional value as well. For consumers, anticake agents may reduce product functionality and performance and, for colored cheeses such as Cheddar, may make the outer shred appearance powdery and discolored. A recent study on consumer perception of commercial Cheddar cheese shreds reported that the powdery coating on Cheddar shreds was a rejecter for consumers, as were shreds that were clumped together (Speight et al., 2019). Meals et al. (2020) also reported that, in a traditional consumer acceptance test, consumers who commented on the powdery appearance of cheese shreds had lower overall liking scores for Cheddar shreds than those who did not. Some consumers even commented that they thought the powdery appearance indicated mold growth (Speight et al., 2019; Meals et al., 2020). These studies suggested that anticake agents for shredded Cheddar cheese may introduce some confusion for consumers.

Additionally, consumers have shown growing skepticism regarding cheese shred anticake agents after a 2016 Bloomberg article reported on cheese products adulterated with "wood shavings" - a reference to cellulose powder application (Mulvany, 2016). Currently, anticake agents are loosely regulated, with no specific limits other than adherence to identity standards of cheese type and generally recognized as safe (GRAS) certification compliance (21CFR170.255; FDA, 2016b). The cheese shreds in the 2016 Bloomberg article were marketed as $100 \%$ Parmesan but did not conform to the FDA standards of identity for Parmesan. With the matching CFR standard of identity for the specific cheese being the only guideline, a company can technically add as much of any GRAS product as they want, as long as it still meets the criteria of milkfat and moisture content for the specific cheese. Many studies and patents have been published on improving the efficiency of anticake agents or investigating alternative anticake agents for shredded cheese (Chappell et al., 2005; Galer et al., 2011; Smith et al., 2014). However, to our knowledge, none have investigated sensory per- ception of anticake agents applied to cheese shreds. The objective of this study was to investigate sensory properties of 3 common anticake mixtures applied at different percentages to Cheddar cheese shreds.

\section{MATERIALS AND METHODS}

\section{Experimental Overview}

Three common anticake agents $(80 \%$ potato starch $/ 20 \%$ cellulose blend, $100 \%$ potato starch, and potato starch/corn starch/calcium sulfate blend) were applied to duplicate lots of Cheddar cheese shreds at 1 , 2, 3, 4, or $5 \%$ (wt/wt). Control Cheddar cheese shreds with no added anticake were included. Sensory properties (appearance, flavor, texture, and hot texture) were documented using a trained sensory panel $(\mathrm{n}=$ 8). Consumer acceptance testing and a home usage test were then conducted on selected cheese shreds. All sensory testing was performed in compliance with the North Carolina State University Institutional Review Board for Human Subjects (Raleigh, NC).

\section{Cheese and Cheese Shreds}

Two lots of full-fat pasteurized milk Cheddar cheese $(291 \mathrm{~kg})$ were produced by a commercial stirred curd cheese manufacture facility in Twin Falls, Idaho. Cheese blocks were manufactured within $24 \mathrm{~h}$ of each other using recombinant chymosin and were cooled using forced-air coolers at $5^{\circ} \mathrm{C}$ for $14 \mathrm{~d}$. These blocks (presented as mean $\pm \mathrm{SD}$ ) were similar in moisture $(38.28 \% \pm 0.03)$, fat in DM $(55.01 \% \pm 0.35)$, salt content $(1.75 \% \pm 0.03)$, and $\mathrm{pH}(5.20 \pm 0.08)$. The blocks were cut into multiple 18.14-kg blocks and shipped to a separate facility located in the Midwestern United States for shredding. Blocks were shredded at $75 \mathrm{~d}$ old.

For shredding, blocks were cut into $5.08-\mathrm{cm}$ cubes, then loaded into a shredder and shredded with a "feather" cut. The resulting shreds were loaded into a tumble drum in 9.07-kg batches, to which the anticake agent was applied. Three common industry anticake agents were applied at $1,2,3,4$, or $5 \%$ (wt/wt) for a total of 15 unique application batches on the duplicate lots of cheese. Anticake agents included an $80 \%$ potato starch $/ 20 \%$ cellulose blend with natamycin (PCELL; Free Flow 206-200, Allied Blending, Keokuk, IA), 100\% potato starch with natamycin (POT; Free Flow 207175, Allied Blending), and a potato starch/corn starch/ calcium sulfate blend with natamycin (PCOR; Free Flow 2036 NG-400, Allied Blending). A 9.07-kg batch of shreds from each block had no anticake agent applied and served as control for a total of 16 treatments in duplicate (15 anticake applications and 1 control with 
no additive $\times 2$ different lots). After anticake application, each batch of shreds was packaged into multiple $2.27-\mathrm{kg}$ plastic bags and heat-sealed with a nitrogen/ carbon dioxide flush to maintain freshness. Packaged Cheddar cheese shreds were shipped on cold gel packs to Raleigh, North Carolina, by overnight carrier. On arrival, cheeses were examined for shipping damage and stored at $5{ }^{\circ} \mathrm{C}$ in the dark until analysis and evaluation. All sensory tests were completed within $21 \mathrm{~d}$ of receipt.

\section{Descriptive Analysis: Visible Powder, Flavor, and Hot and Cold Texture}

Descriptive sensory analysis of visible powder, flavor, cold texture, and hot texture properties of cheese shreds were conducted by a trained panel $(\mathrm{n}=8,5$ women, 3 men, ages 23-54 yr). All data were collected using Compusense Cloud software (Compusense, Guelph, Canada). Visible powder, flavor, cold texture, and hot texture were evaluated in separate sessions.

For visible powder appearance, trained panelists evaluated the appearance of the cheese shreds. A 0- to 10 -point intensity scale was used, where 0 represented no visible anticake agent and 10 represented all surfaces of the Cheddar cheese shreds visibly coated in anticake agent. Approximately $15 \mathrm{~g}$ of cheese shreds were presented in 100-mm o.d. $\times 15$-mm Petri dishes (Kimble-Chase Solutions, Vineland, NJ) against a black polyethylene tablecloth (Spritz, Minneapolis, MN) under fluorescent lights (F32T8/ADV841/XEW, Phillips, Amsterdam, the Netherlands) at 660 lx. Samples were blinded with random 3-digit codes and were evaluated at $4^{\circ} \mathrm{C}$ to prevent condensation from affecting the visual appearance. Each panelist evaluated each cheese shred in duplicate over the course of 4 sessions. During each session, panelists were presented with a random selection of 8 (of a possible 16) shred samples.

For flavor analysis, trained panelists evaluated the cheeses using an established Cheddar cheese flavor lexicon (Drake et al., 2001) and a 0- to 15-point universal intensity scale consistent with the Spectrum method (Lawless and Heymann, 2010). Each panelist had at least $150 \mathrm{~h}$ of experience with descriptive analysis of cheese flavors. Cheddar cheese shreds (approx. $15 \mathrm{~g}$ ) were served in lidded 120 -mL clear plastic soufflé cups (Dart Container, Mason, MI) with random 3-digit blinding codes, and samples were evaluated at $15^{\circ} \mathrm{C}$. Each panelist evaluated each cheese shred in duplicate over the course of 8 sessions. Within each session, panelists were presented a random presentation of 4 shred samples. Between sample evaluations, a 5-min rest time was enforced, during which time panelists were encouraged to cleanse their palates with available deionized water and unsalted crackers.
For hot and cold texture evaluations, trained panelists evaluated the cheese shreds using a 0 - to 15 -point product-specific scale. The cheese texture lexicons developed by Drake et al. (1999), Gwartney et al. (2002), Asato (2003), Brown et al. (2003), and Rogers et al. (2009) were used, but terms were modified to accommodate evaluations specific to cheese shreds at different temperatures (Table 1). Hot and cold texture were evaluated in different sessions. Approximately $15 \mathrm{~g}$ of cold cheese shreds were served at $4^{\circ} \mathrm{C}$ in lidded $120-\mathrm{mL}$ soufflé cups (Dart Container, Mason, MI) with 3-digit blinding codes. For hot texture, approximately $15 \mathrm{~g}$ of each Cheddar cheese shred was baked in clean metal desiccation dishes $7.62 \mathrm{~cm}$ in diameter (VWR International, Radnor, PA) in a conventional oven set to $230^{\circ} \mathrm{C}$ for $4 \mathrm{~min}$. Cheese texture was evaluated within $1 \mathrm{~min}$ of being removed from the oven. For both cold and hot texture evaluations, each panelist evaluated each cheese shred in duplicate over the course of 4 sessions. Within each session, panelists were presented a random presentation of 8 shred samples. Between sample evaluations, a 5-min rest time was enforced, during which time panelists were encouraged to cleanse their palates with available deionized water and unsalted crackers.

\section{Consumer Testing}

Consumer acceptance of the Cheddar shreds was evaluated with 3 separate tests: a visual test, a melted test, and a home usage test (HUT). For all consumer tests, the Cheddar cheese shred replicate batches were commingled, as descriptive analysis showed no differences $(P>0.05)$ across lots. Online questionnaires developed using Compusense Cloud software were used for data collection.

\section{Cold Appearance Liking}

Consumer acceptance testing was conducted to investigate consumer visual perception of anticake agents on cold Cheddar cheese shreds. Based upon descriptive analysis results, 13 shreds were selected, as these were found to be significantly different in visible appearance by trained panelists $(P<0.05)$. Self-reported Cheddar cheese shred consumers $(\mathrm{n}=150)$ were recruited the day of the test through an email sent to an online database of 11,000 individuals maintained by North Carolina State University. Recruitment was open to anyone $>18$ yr who was a self-reported Cheddar cheese shred consumer. Consumer acceptance testing was performed on a single day, with each consumer evaluating visual appearance of 10 of 13 samples in a randomized balanced incomplete block (BIB) design. When consumers arrived for their test, they were 
provided an Apple iPad (Apple Inc., Cupertino, CA) loaded with Compusense Cloud software and received verbal instruction on testing procedures. Consumers were asked to evaluate overall appearance liking, color liking, purchase intent, color just-about-right (JAR; from light to dark and yellow to orange), and check-allthat-apply (CATA) visual Cheddar cheese attributes for each sample presented. Upon completion of the entire test, consumers were compensated with a $\$ 5$ gift card to a local store.

Each Cheddar cheese shred sample consisted of approximately $100 \mathrm{~g}$ of cheese shreds in $15.24 \mathrm{~cm} \times 20.32$ $\mathrm{cm}$ clear low-density polyethylene bags (Uline, Pleasant Prairie, WI) with random 3-digit blinding codes. The testing room was cleared to allow panelists to walk around the room comfortably. Three tables $(152.4 \times$ $45.72 \mathrm{~cm}$ ) with black polyethylene tablecloths (Spritz) were set up around the testing room's perimeter. A sample bag of each of the 13 cheeses in the test was displayed at each table under fluorescent lights (F32T8/ ADV841/XEW, Phillips) at $660 \mathrm{~lx}$. Panelists were allowed to stand to assess samples and were informed that they could pick up the samples to more closely examine the bagged products. Samples were replaced every $30 \mathrm{~min}$ to prevent condensation from affecting the visual appearance. Shreds were evaluated monadically using a BIB design, wherein consumers evaluated 10 out of the 13 samples.

Liking questions were scored on a 9-point hedonic scale where $1=$ dislike extremely and $9=$ like extremely. The JAR questions used a 5-point scale where 1 or $2=$ too little, $3=\mathrm{JAR}$, and 4 or $5=$ too much. Purchase intent was scored on a 5 -point scale where 1 or $2=$ would not buy, $3=$ may or may not buy, and 4 or $5=$ would buy. The CATA Cheddar cheese shred attribute question included soft, hard, dry/crumbly, rubbery, short shreds, long shreds, thick shreds, thin shreds, orange, yellow, white, powdery, and powderless.

\section{Melted Consumer Test}

Ten cheese shreds were selected for melted cheese consumer acceptance testing. Shreds selected were control (no anticake agent added), and applications of 1,3 , or $5 \%$ (wt/wt) of each of the 3 anticake agents. These samples were selected to determine liking of each anticake agent at a high, medium, and low application. The control was added to compare against the shreds with anticake agents added. Self-reported Cheddar cheese shred consumers $(n=250)$ were recruited to participate in sensory testing of melted Cheddar cheese shreds using a survey launched into an online database of 11,000 individuals maintained by North Carolina State University. All consumers were primary shoppers with an annual household income $>\$ 15,000$ who purchased Cheddar cheese shreds at least once a month. Of the consumers recruited, sex was split equally between men and women, and income was split equally among the recruitment income ranges to achieve a broad range of consumers. Consumer acceptance testing was performed over $2 \mathrm{~d}$, with each consumer completing the test in a single seating. As consumers arrived for their test, they first presented photo identification to ensure that the consumers who participated were the consumers screened for the test. Consumers were then verbally instructed on the testing method. Consumers then evaluated 4 of the 10 samples in a randomized BIB design during their seating. Panelists were compensated with a $\$ 15$ gift card to a local store upon completion of the entire test.

Table 1. Trained panel cheese shred texture attributes (adapted from Drake et al., 1999; Gwartney et al., 2002; Asato, 2003; Brown et al., 2003; Rogers et al., 2009)

\begin{tabular}{|c|c|}
\hline Attribute & Definition \\
\hline \multicolumn{2}{|l|}{ Cold visual appearance } \\
\hline Visible powder & $\begin{array}{l}\text { Amount of visible powder on the shreds, where } 0=\text { no visible powder and } 10=\text { every shred surface is coated } \\
\text { in powder }\end{array}$ \\
\hline \multicolumn{2}{|r|}{ P } \\
\hline Hand cohesiveness & Degree to which the sample forms a mass and does not break apart after compressing $30 \%$ for $5 \mathrm{~s}$ then release \\
\hline \multicolumn{2}{|r|}{ 20 - } \\
\hline Visible surface moisture & Amount of oil resting on the surface \\
\hline Meltedness & Homogeneity of the sample (higher number = more melted) \\
\hline Stretchability & Length the melted cheese will stretch with a plastic fork within a minute of oven removal \\
\hline Oiliness to lips & Amount of oil felt on lips \\
\hline Hardness & Amount of force required to bite between molars during first bite \\
\hline Tackiness & Amount of force required to pull teeth apart after first bite \\
\hline Cohesiveness & Degree to which the sample forms a mass and does not break apart after 5 chews \\
\hline Adhesiveness & Degree to which the sample sticks to any of the mouth surfaces during mastication \\
\hline Oiliness & Amount of oil or any coating perceived in the mouth \\
\hline Smoothness of mass & Lack of gritty or grainy particles perceived in the mass while chewing \\
\hline Mouthcoat & Amount of any mouthcoating (particles, oil, moisture) remaining after swallowing or expectorating \\
\hline
\end{tabular}




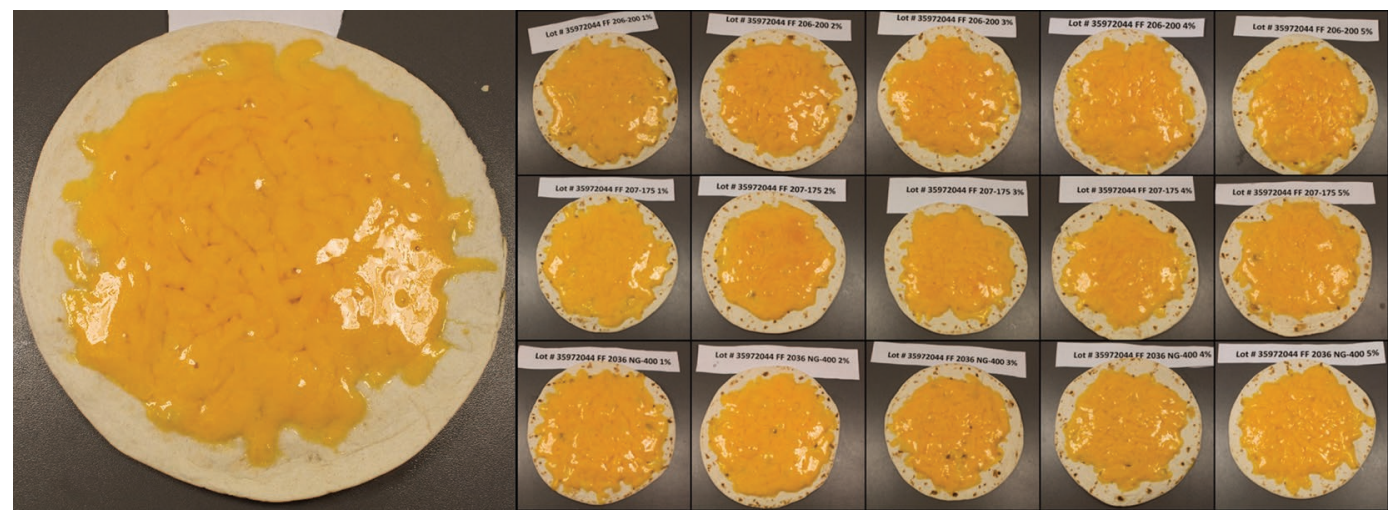

Figure 1. Test cook of $40 \mathrm{~g}$ of control (leftmost picture; no anticake) and test cheese shreds baked at $204^{\circ} \mathrm{C}$ for $70 \mathrm{~s}$ in an impinger oven. Top row: PCELL, $80 \%$ potato starch/20\% cellulose blend; middle row: POT, 100\% potato starch; bottom row: PCOR, potato starch/corn starch/ calcium sulfate blend; 1 to $5 \%$ (wt/wt) application rate, left to right.

Samples were presented as $20 \mathrm{~g}$ of cheese shreds melted onto an open-face $15.24-\mathrm{cm}$ flour tortilla, cut in half (UPC: 073731008300, Mission Foods, Irving, TX). Cheese was melted using a Lincoln Impinger Oven model 1301 (Fort Wayne, IN) set to $204^{\circ} \mathrm{C}\left(400^{\circ} \mathrm{F}\right)$ with a belt speed to allow for $70 \mathrm{~s}$ of heating. This length and speed were determined before consumer testing by using the control $(0 \%)$ cheese and adjusting time and temperature to allow for a quick and full melt without burning (Figure 1). For the consumer test, panelists were served half tortillas, so the tortillas were sliced in half before testing, and only $20 \mathrm{~g}$ of cheese were melted. Samples were prepared as consumers needed each sample, to ensure uniform serving temperature throughout testing.

Once a sample was presented, consumers were first asked to evaluate overall appearance liking, visible melt liking, and visible melt JAR. After evaluating appearance, consumers were instructed to consume several bites before evaluating cheese flavor and texture. Questions including overall liking, overall flavor liking, texture liking, stretch liking, stretchiness JAR, chewiness JAR, purchase intent, CATA melted Cheddar cheese shred texture and appearance attributes, and purchase intent were subsequently asked. A 3-min rest was enforced between samples, and during this time consumers were asked to cleanse their palates using provided unsalted crackers and deionized water.

Liking, JAR, and purchase intent questions were scaled as described previously. The CATA Cheddar cheese shred question contained different attributes than the cold visual central location test (CLT), with the list of terms including soft, hard, dry, burned, rubbery, melted, cohesive, stringy, greasy, oily, smooth, stretchy, stiff, breakable, and the option of "other" with a comment box to clarify this selection. The CATA terms were adapted from descriptive lexicons for cheese texture (Asato, 2003; Brown et al., 2003).

\section{Home Usage Test}

Seven cheeses were selected for HUT. Shreds selected were control (no anticake agent added), and the 1 and $5 \%$ (wt/wt) applications of each of the 3 anticake agents. These samples were selected to determine liking of each anticake agent at a high and low application during home usage. The control was included to compare against the shreds with anticake agents. Consumers $(\mathrm{n}=113)$ were randomly recruited from the melted cheese test. Those who chose to participate received a $38-\times 30.5-\times 15.2-\mathrm{cm}$ thermal bag (Uline) with three $15.24-\mathrm{cm} \times 20.32-\mathrm{cm}$ clear low-density polyethylene bags (Uline) labeled with random 3-digit blinding codes, with each containing approximately 150 $\mathrm{g}$ of shreds. A $12.7-\times 7-\times 2$-cm single-use ice pack (Uline) was added, to maintain refrigerated temperature during transport home. The freezer bag included an information sheet that contained test directions, the consumer's individual login credentials, and a link to the test website. Consumers evaluated samples in a randomized BIB design, evaluating 3 of the 7 shred samples using an online questionnaire designed for sensory data capture (Compusense). Consumers were allowed $5 \mathrm{~d}$ to complete the test. Panelists were able to complete sample evaluations at their own pace, but a 4-h enforced wait period was programmed between each sample evaluation to discourage "click-through" responses. Consumers who completed the entire test received a $\$ 15$ gift card to a local store.

For each sample, consumers first evaluated the shreds before consuming, answering an overall appearance liking question, an open-ended appearance comment, and 
expectations question. After evaluation of appearance, consumers prepared a meal or snack with the shreds and evaluated overall appearance liking with an open-ended appearance comment, flavor liking, and texture liking. If consumers prepared a hot snack, they were asked additional questions regarding melt liking, melt JAR, stretch liking, and chewiness JAR. Each consumer then answered questions for purchase intent and similarity to ideal Cheddar shreds. Consumers were required to take pictures of their meal or snack before and after the evaluation, as a way to ensure that instructions were followed. Liking, JAR, and purchase intent questions were scaled as described previously. Expectation was scored on a 5 -point scale where 1 and $2=$ does not meet expectations, $3=$ maybe/maybe not meets expectations, 4 and $5=$ meets expectations. Similarity to ideal Cheddar shreds was scored on a 5-point scale where 1 and $2=$ not similar, $3=$ maybe/maybe not similar, 4 and $5=$ similar.

\section{Statistical Analysis}

Statistical analyses were conducted using XLSTAT software (version 2017; Addinsoft, New York, NY). Descriptive analysis and consumers liking scores were analyzed by 2-way ANOVA (percent application $\times$ anticake agent) with Fisher's least significant difference test $(\alpha=0.05)$. One-way ANOVA with Fisher's least significant difference test $(\alpha=0.05)$ was also used, where appropriate, to compare with the control. Consumer JAR scores were evaluated by chi-squared analysis, and purchase intent was evaluated using the Kruskal-Wallis test with Dunn's post hoc test.

\section{RESULTS}

\section{Descriptive Analysis}

Cheese shreds were characterized by high intensities of cooked/milky and whey flavors consistent with a characteristic young Cheddar flavor profile (Drake et al., 2001, 2008; results not shown). Flavor attributes of shreds were consistent $(P>0.05)$ and unaffected by anticake agent except for the flavor attribute plastic/ chemical. Cheese shreds with $5 \%$ (wt/wt) of any of the 3 anticake agents had low but distinct plastic/chemical flavor (mean $=0.8 \pm 0.2,0$ - to 15 -point intensity scale).

Distinguishing texture differences related to the presence and application percent of anticake agents were documented by the trained panel $(P<0.05$; Table 2$)$. No significant differences were detected between the lots of cheese $(P>0.05)$. Visual analysis of the cheese shreds demonstrated that at $1 \%$ (wt/wt) none of the anticake agents had visible powder on the Cheddar shreds. The POT anticake agent was least affected by application rate. Visible powder for all anticake agents increased at 2\% (wt/wt) application to shreds $(P<$ 0.05). However, visible powder on shreds with PCELL or PCOR increased sharply at 3 and $4 \%$ (wt/wt), respectively $(P<0.05)$, compared with shreds with POT.

Hand cohesiveness results were similar to those of visible powder, in that the shreds with added POT were least affected by application rate. When each treatment was compared with the control with no anticake, POT was at parity with the control at 1 and $2 \%$ (wt/wt; $P$ $>0.05$; results not shown). Two-way ANOVA between anticake type and percent application showed that both PCELL and PCOR reduced hand cohesiveness more than POT $(P<0.05)$. As expected, as application percentage increased, hand cohesiveness decreased for all the anticake agents $(P<0.05)$.

Anticake agents and percent application affected some hot cheese texture terms (Table 2). Firmness, tackiness, oiliness (in-mouth), and mouthcoating were not affected by anticake agents $(P>0.05$; results not shown). Compared with the control (no anticake agent), anticake agent and percent application had variable effects $(P<0.05)$ for visible oil, oiliness to lips, cohesiveness, smoothness, and stretch, with some shreds being unaffected by anticake agent up to $3 \%$ (wt/wt) in these attributes. Shreds with POT had lower initial visible oil at 1\% (wt/wt) application than shreds with PCOR or PCELL, but visible oil was less affected by application rate compared with shreds with PCOR or PCELL. Shreds with PCOR, across the application rates evaluated, were least affected of the 3 anticake agents in oiliness to the lips $(P<0.05)$ compared with shreds with POT and PCELL. For smoothness of mass, shreds with PCOR at $5 \%$ (wt/wt) scored lowest in intensity $(P<$ 0.05 ). This anticake agent was a potato starch with calcium sulfate and corn starch blend, so it could be inferred that these anticake additives may contribute to a rougher mouthfeel, perhaps due to differences in particle size or shape. Additional work would be needed to confirm this hypothesis. In stretch descriptive analysis, shreds were unaffected by the anticake agents $(P>0.05)$ until 3\% (wt/wt) for PCOR and POT and 2\% (wt/ wt) for PCELL. At percentage applications $>2 \%$ (wt/ wt), shreds with PCOR were least affected by anticake agents, and shreds with PCELL were most affected $(P$ $<0.05)$. For cohesiveness evaluation, shreds with 1 and $2 \%$ (wt/wt) PCELL had the highest intensities, but at $5 \%$ (wt/wt), shreds with PCELL had the lowest scores $(P<0.05)$. Visual meltedness was affected by percent application, but not by specific anticake agent. Shreds with $>2 \%$ (wt/wt) anticake agent received lower visual meltedness scores $(P<0.05)$. 
Differences in descriptive sensory data were explained with 2 principal components $(87 \%$ of the variability; Figure 2). Based on factor loadings, principal component $1(75 \%)$ differentiated shreds by hand cohesiveness, visual meltedness, stretch, cohesiveness, oiliness to lips (positively loading), and plastic flavor and visible powder (negatively loading). Principal component 2 differentiated shreds by visible oil (negatively loading) and smoothness of mass (positively loading). The biplot illustrated the effects on sensory attributes for each anticake agent as percentage application increased. The control cheese shreds were characterized with the highest smoothness of mass and hand cohesiveness, visual meltedness, stretch, cohesiveness, oiliness to lips, and visible oil. As percent anticake application increased, shreds become less similar to the control with reduced smoothness of mass, hand cohesiveness, visual meltedness, stretch, cohesiveness, oiliness to lips, and visible oil, and increased visible powder and plastic flavor. As previously stated, shreds with POT were correlated with reduced visible powder and increased smoothness of mass hand cohesiveness, but had reduced oiliness to lips, cohesiveness, and visible oil. Shreds with PCELL or PCOR, however, had increased visible powder and reduced smoothness of mass and hand cohesiveness, but shreds with either of these 2 anticake agents had increased stretch, oiliness to lips, and visible oil compared with shreds with POT.

\section{Consumer Testing: Cold Appearance Liking}

Consumer evaluations of cold shred appearance were consistent with results from the trained panel (Table 3 ). Cheese shreds with POT anticake were least influenced by percent application, whereas shreds with PCELL or PCOR decreased in liking at $>4 \%$ (wt/wt) application. Purchase intent showed similar findings, in that the control (no anticake) shreds were at parity with shreds with all applications of POT and shreds with applications of 2 to $3 \%$ (wt/wt) for the other 2

Table 2. Trained panel evaluation of visible powder, cold texture, and hot texture attributes for Cheddar cheese shreds with different anticake applications and application rates $(\mathrm{ND}=$ not detected)

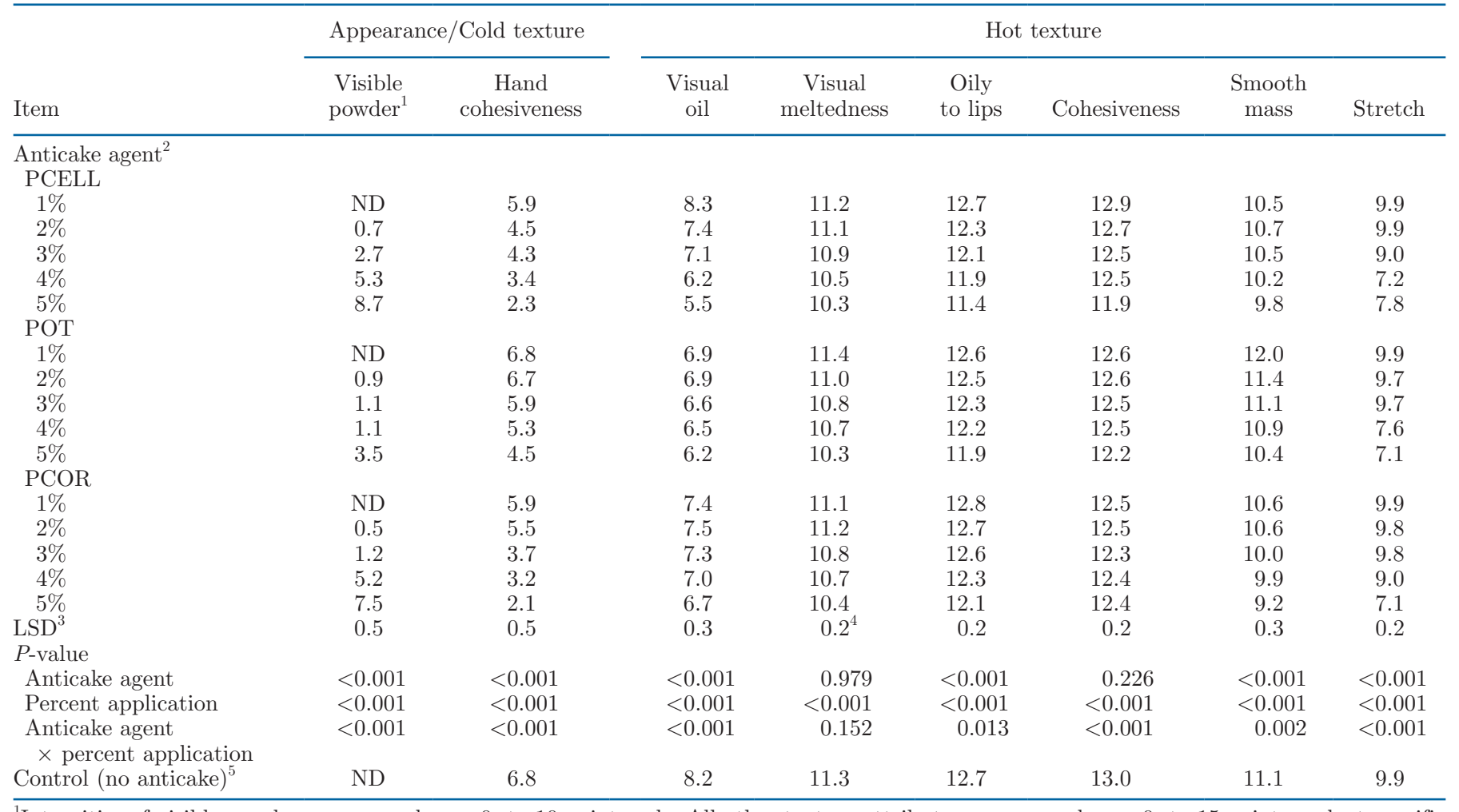

${ }^{1}$ Intensities of visible powder were scored on a 0- to 10-point scale. All other texture attributes were scored on a 0 - to 15 -point product-specific scale.

${ }^{2}$ PCELL $=80 \%$ potato starch $/ 20 \%$ cellulose blend; POT $=100 \%$ potato starch; PCOR $=$ potato starch $/$ corn starch $/$ calcium sulfate blend . Percent refers to amount of anticake added (wt/wt) to shreds.

${ }^{3}$ Means in a column that differ by LSD are significantly different $(P<0.05)$.

${ }^{4} \mathrm{LSD}$ for visual meltedness is for percent application main effect.

${ }^{5}$ Control was excluded from the 2-way model. 
Table 3. Consumer liking means for cold Cheddar cheese shred appearance evaluation $^{1}(\mathrm{n}=110$ through each shred $)$

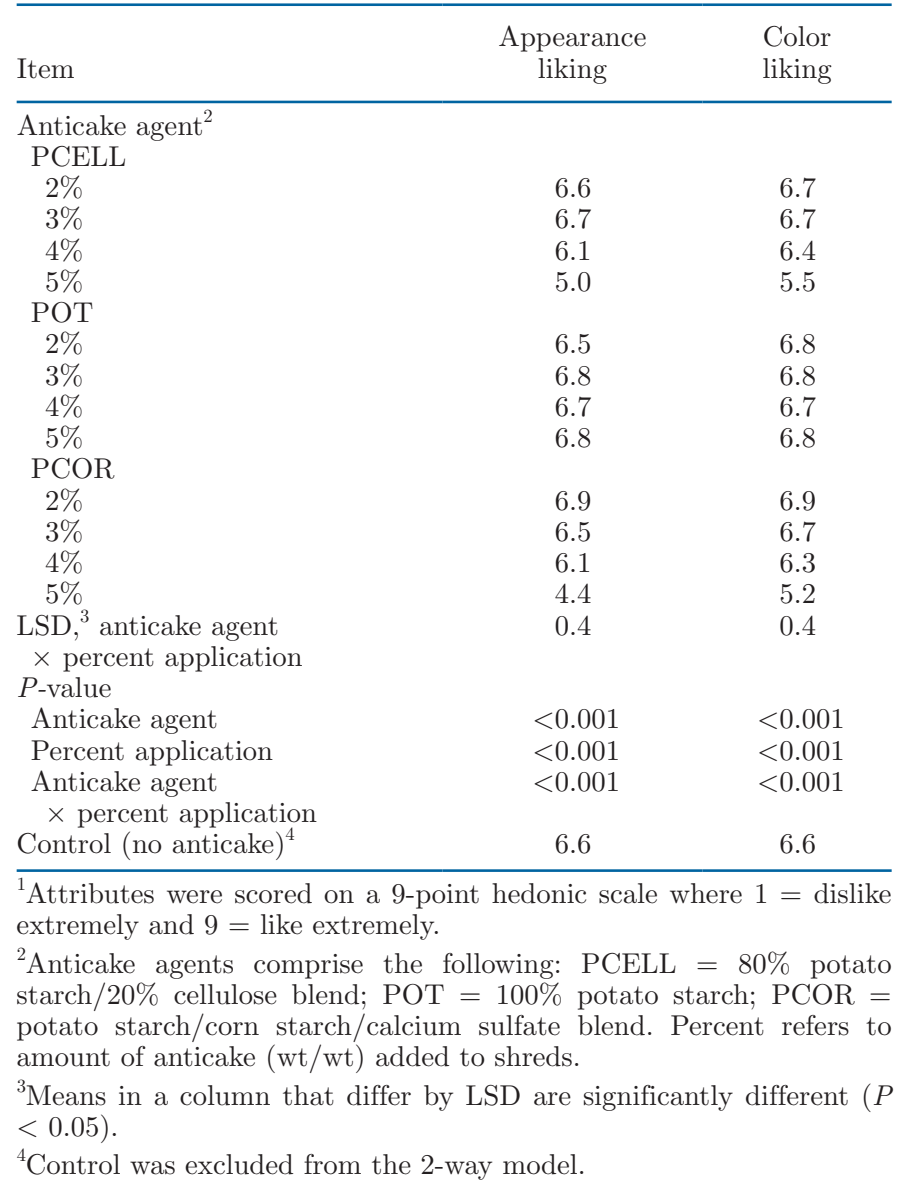

anticakes $(P<0.05$; results not shown). Analysis of CATA consumer selections showed that all shreds with POT received selection frequencies at parity $(P>0.05)$ with the control (no anticake; Table 4). Specifically, for powdery and powderless attributes, shreds with POT were similar to the control $(P>0.05)$. Shreds with PCELL and PCOR both differed from control in selection frequencies of powdery and powderless $(P<0.05)$, again suggesting that these anticake agents had greater effects on consumer liking than shreds with POT under cold (as-is) conditions.

\section{Consumer Testing: Melted Liking}

Increased percent application had a negative effect on overall liking, cheese flavor liking, cheese texture liking, and stretch liking $(P<0.05)$ regardless of anticake agent (Table 5, Figure 3). Interactions between anticake type and percent application were significant only for the visual liking questions: overall appearance and melt. Here, shreds with POT had the largest influence on liking and at $5 \%$ (wt/wt) received the lowest liking scores in those categories $(P<0.05$; Figure 4$)$. Shreds with the 3 anticake agents were penalized at 3 and $5 \%$ (wt/wt) for not appearing melted enough (Table 6). Trained panelists also documented reduced visible oil and visual meltedness with increased anticake application, regardless of anticake type (Table 2). Interestingly, the control shreds and PCOR at 1\% (wt) wt) were penalized for being "too stretchy." This may

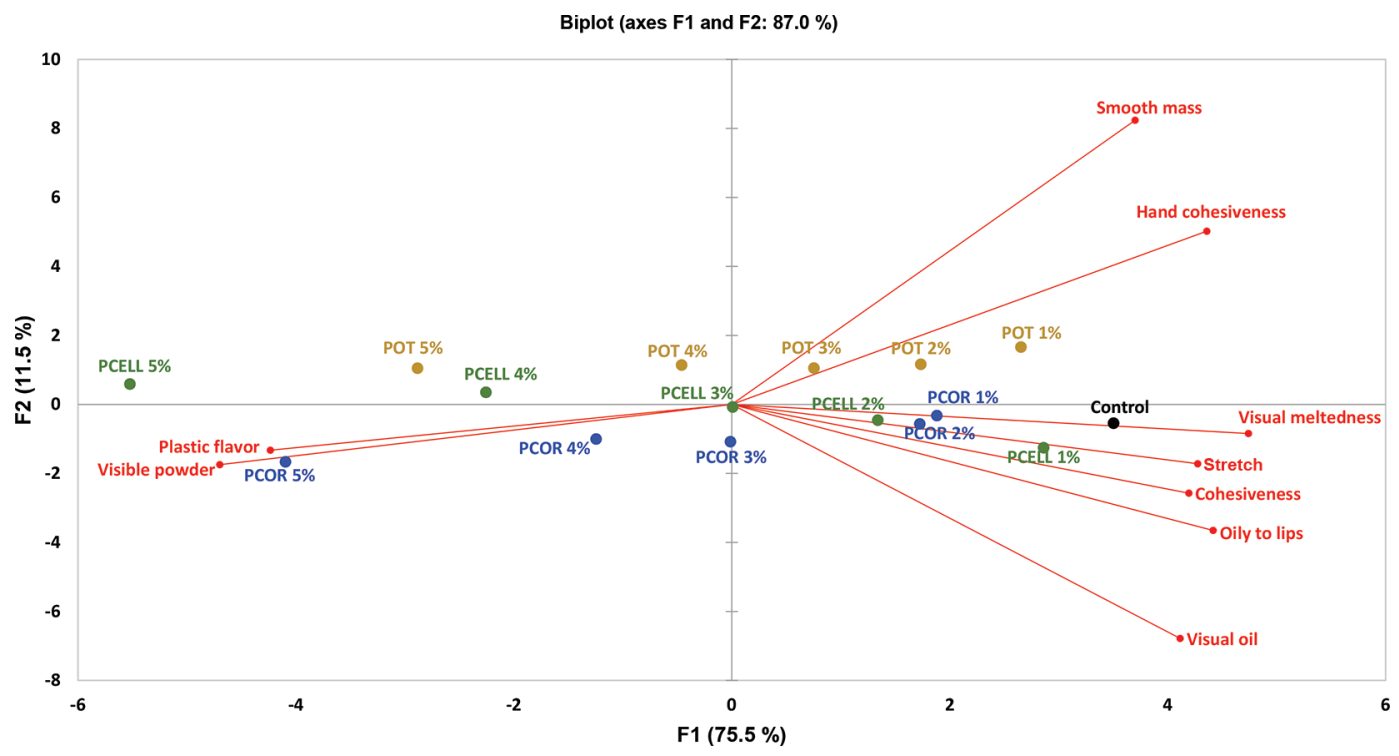

Figure 2. Principal component biplot of significant Cheddar cheese shred trained panel evaluation of flavor, hot texture, cold texture, and visible powder attributes for different anticake agents at applications of 1 to $5 \%$ (wt/wt). Anticake agents comprised the following: PCELL = $80 \%$ potato starch $/ 20 \%$ cellulose blend; POT $=100 \%$ potato starch; PCOR $=$ potato starch/corn starch/calcium sulfate blend. 
Meals et al.: ANTICAKE AGENTS IN CHEDDAR CHEESE

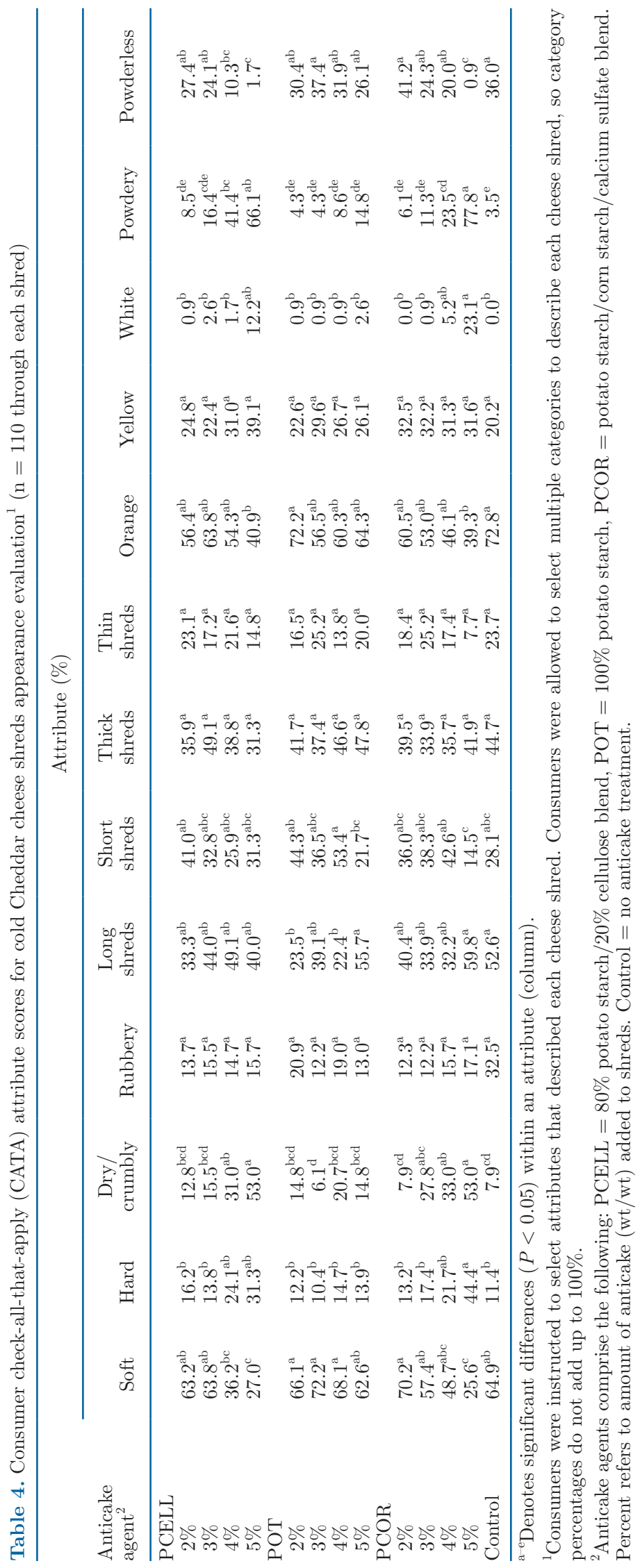


suggest that consumers are not used to the extent of stretch from young cheeses without anticake. Cheese shreds with each anticake at $5 \%$ (wt/wt) were penalized for not being stretchy enough.

\section{Home Usage Test}

Cold appearance and in-food appearance were the only liking questions that were distinct among the shreds tested by HUT $(P<0.05$; Table 7$)$. Responses for all other liking questions were at parity $(P>0.05)$. Analysis of the effects of percent application and anticake type showed no interaction effect for any of the liking questions, and that percent application was a significant effect for cold appearance and in-food appearance liking $(P<0.05)$. Cheese shreds with $5 \%$ (wt/ wt) application of any of the 3 anticake agents had lower cold appearance liking scores $(\mathrm{x}=6.7$ vs. 7.5 on a 1- to 9-point scale) and in-food appearance liking scores $(\mathrm{x}=7.6$ vs. 7.1 on a 1 - to 9 -point scale) than $1 \%$ (wt/wt) application $(P<0.05)$, and this result was consistent with the previous cold appearance consumer liking and trained panel results.

When each shred was individually compared with the control, all 3 cheese shreds with $5 \%$ (wt/wt) application of any of the 3 anticake agents scored lower than the control for cold appearance liking $(P<0.05)$. Of those, shreds with PCOR or PCELL scored significantly lower than shreds with POT $(P<0.05)$. Shreds at $1 \%$ (wt/wt) application were at parity with the control $(P>0.05)$. For in-food appearance liking, shreds with PCELL at 5\% (wt/wt) were the only shreds that scored lower than the control with no anticake added $(P<0.05)$. Analysis of the cold expectations, purchase intent, and ideal cheese questions again reiterated that anticake influenced initial consumer expectations. All shreds scored at parity for postconsumption purchase intent and similarity to ideal cheese questions $(P>$ 0.05 ; mean $=3.9 \pm 0.1,4.0 \pm 0.1$, respectively, 0 - to 5 -point scale; results not shown).

\section{DISCUSSION}

Descriptive sensory texture profiles for Cheddar shreds with 3 different anticake agents were affected at increasing percent applications, and effect varied with anticake agent. Akins (2002) tested mozzarella shreds with $2 \%$ (wt/wt) applications of anticake agents containing varying amounts of cellulose and potato starch. That study reported that anticake agents with potato starch had reduced free oil formation (visual oil/

Table 5. Consumer liking means for melted Cheddar cheese shred central location test ${ }^{1}(\mathrm{n}=100$ through each shred)

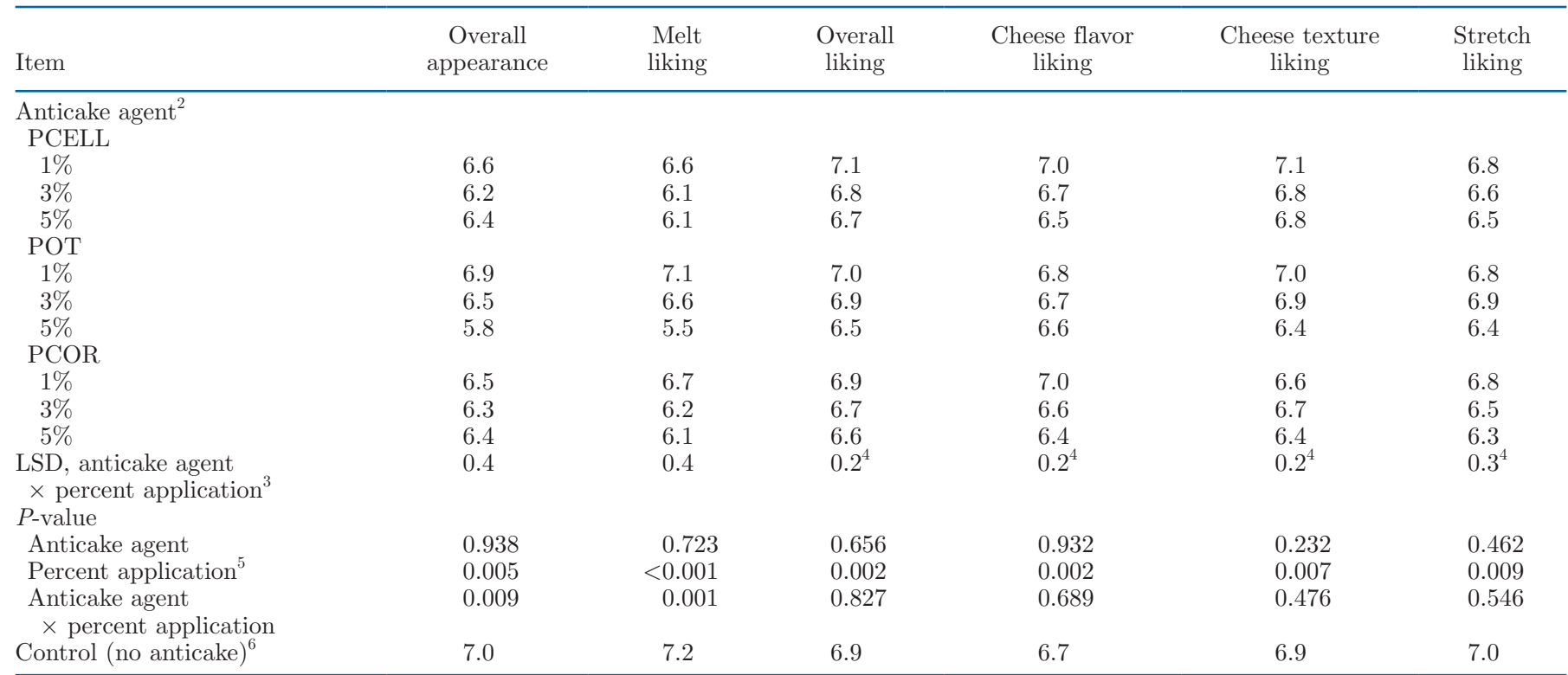

\footnotetext{
${ }^{1}$ Attributes were scored on a 9-point hedonic scale, where $1=$ dislike extremely and $9=$ like extremely.

${ }^{2}$ Anticake agents comprise the following: PCELL $=80 \%$ potato starch $/ 20 \%$ cellulose blend; POT $=100 \%$ potato starch; PCOR $=$ potato starch $/$ corn starch/calcium sulfate blend. Percent refers to amount of anticake (wt/wt) added to shreds.

${ }^{3} \mathrm{LSD}$ for the interaction of anticake agent $\times$ percent application for overall appearance and melt liking.

${ }^{4}$ LSD for overall liking, cheese flavor liking, cheese texture liking, and stretch liking are for percent application main effect.

${ }^{5}$ Percent application main effects are shown in Figure 3.

${ }^{6}$ Control was excluded from the 2-way model.
} 


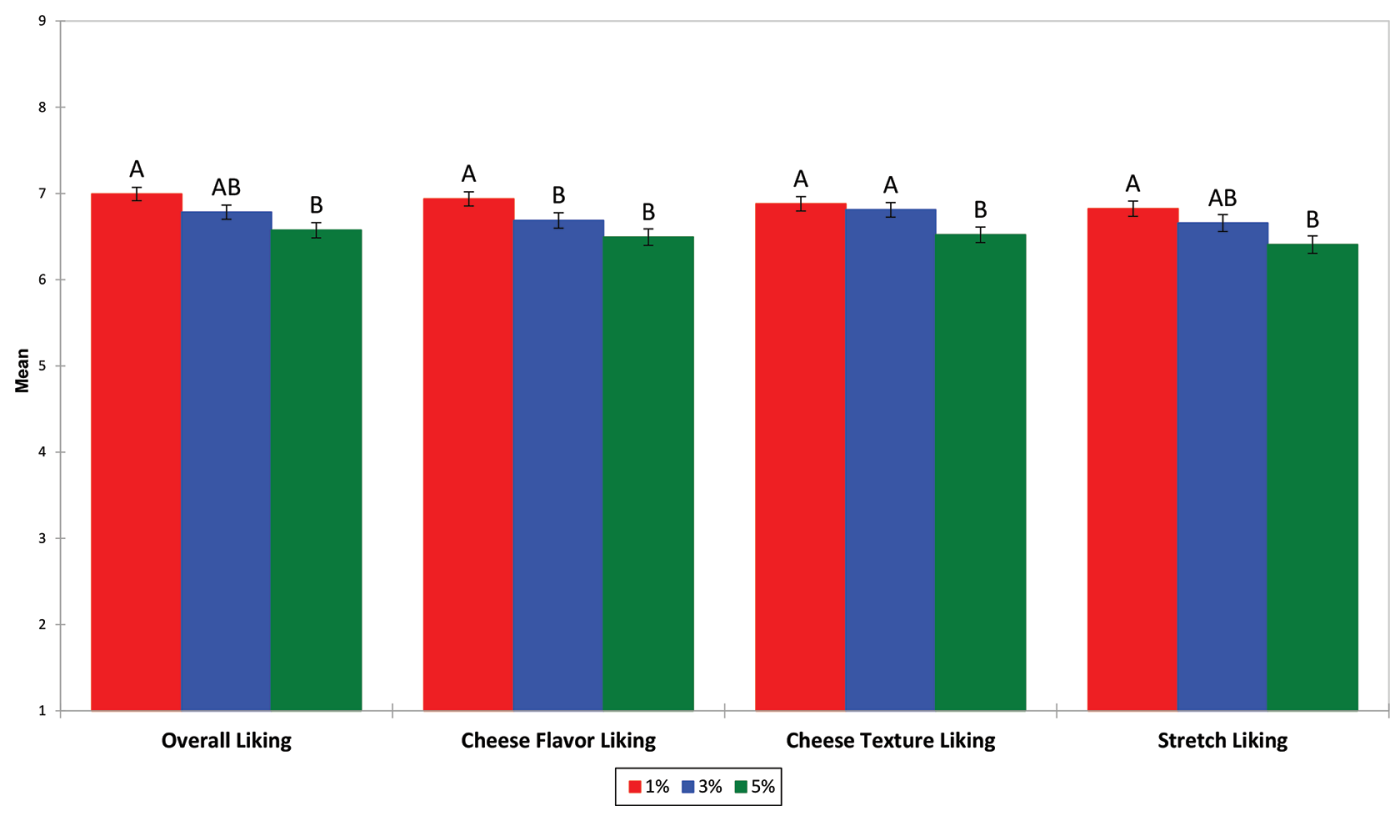

Figure 3. Consumer liking means for melted Cheddar cheese shreds averaged across anticake agent at 1, 3, and 5\% application (wt/wt); n $=300$ through each percent application. Liking scores were pooled across anticake agents because anticake agent percent interactions were not significant $(P>0.05)$. Different uppercase letters within liking questions signify significant differences between means $(P<0.05)$.

sheen) when melted compared with pure cellulose and primarily cellulose blends. Although that study tested applications of anticake agents only at $2 \%$ (wt/wt) for mozzarella, the reduction of free oil for blends with predominantly potato starch was similar to the findings of the current study. Shreds with POT received the lowest scores in visible oil when melted by trained panelists. Visible oil and melt also decreased with in- creased anticake application with all 3 anticake agents (Table 2), consistent with decreases in melt liking by consumers (Table 5). Visible or free oil with melted cheese may be a positive and a negative factor to consumers (e.g., some is good, but too much is bad), but in the current study, visible oil and degree of melt are intertwined (Table 2). At 1 and 3\% (wt/wt) application, the melt liking of shreds with POT was preferred

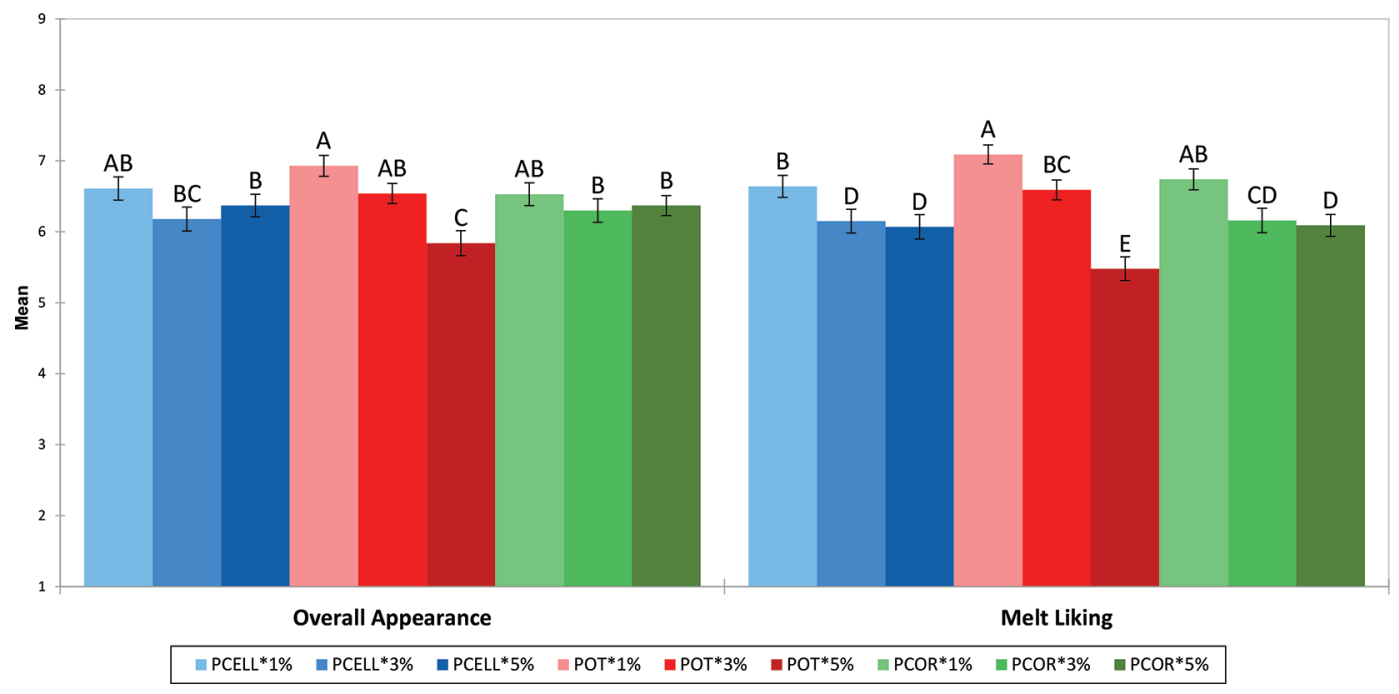

Figure 4. Consumer liking means for melted Cheddar cheese shreds for each anticake agent at 1,3 , or $5 \%$ (wt/wt) application; $\mathrm{n}=100$ through each shred. Anticake agents comprised the following: PCELL $=80 \%$ potato starch $/ 20 \%$ cellulose blend; POT $=100 \%$ potato starch; $\mathrm{PCOR}=$ potato starch/corn starch/calcium sulfate blend. Different uppercase letters within liking questions signify significant differences between means $(P<0.05)$. 
over shreds with PCELL (Table 5). Trained panelists also confirmed that at 1 and $3 \%$ (wt/wt), shreds with POT had the least visible oil. We can infer from the current results that consumers prefer a complete visible melt with Cheddar shreds and that, within acceptable visible melt, less free oil is preferred over more free oil. Additional work focused on visible oil and melt would be needed to identify at what specific point visible oil and degree of melt are not acceptable. Akins (2002) also reported that at $2 \%(\mathrm{wt} / \mathrm{wt})$ anticake application, the blend composition did not affect mozzarella stretch or meltability. This finding is also consistent with the current study's finding, that anticake agents were similar in sensory properties until 3\% (wt/wt) application. Akins (2002) did not investigate consumer perception of the mozzarella shreds with differing anticake agents. In the current study, the trained panel sensory evaluation documented specific texture differences between type of anticake agent applied to cheese shreds, but these differences may not influence consumer liking and purchase intent.

In the current study, visible appearance of cheese shreds was the most important parameter for consumers. Percent application was a main effect for all liking questions in the cold visual and melted consumer tests and for the cold appearance and in-food appearance liking questions in HUT. As expected, as percent application increased, liking decreased. Unlike the melted consumer CLT, where percent application effects for consumption and use liking questions were documented, the HUT consumption liking scores (flavor, texture, melt, stretch) were not affected by anticake agent or percent application, with all shreds at parity at $95 \%$ confidence. Previous studies have documented that consumers tend to be less critical in HUT conditions, both assigning higher liking scores and noting fewer differences in liking (Boutrolle et al., 2005, 2007; Sosa et al., 2008; Sveinsdóttir et al., 2010; Zhang et al., 2020). In the current study, HUT consumers could evaluate the shreds hot or cold, and this might also explain the differences between the CLT and the HUT.

The type of anticake agent affected visual liking questions in all 3 consumer tests. Shreds with POT received higher scores for overall appearance and color liking than the other anticake agents in the CLT with cold shreds $(P<0.05)$. Shreds with POT at $5 \%$ (wt/ wt) also received the lowest liking scores for overall appearance when hot and melt liking $(P<0.05)$. The HUT scores for visual liking were less affected by the type of anticake agent. The effect of anticake agent was significant for cold appearance and in-food appearance liking near $90 \%$ confidence, but not at $95 \%$. The differences in significance between consumer tests may be the difference between CLT and HUT methods as well

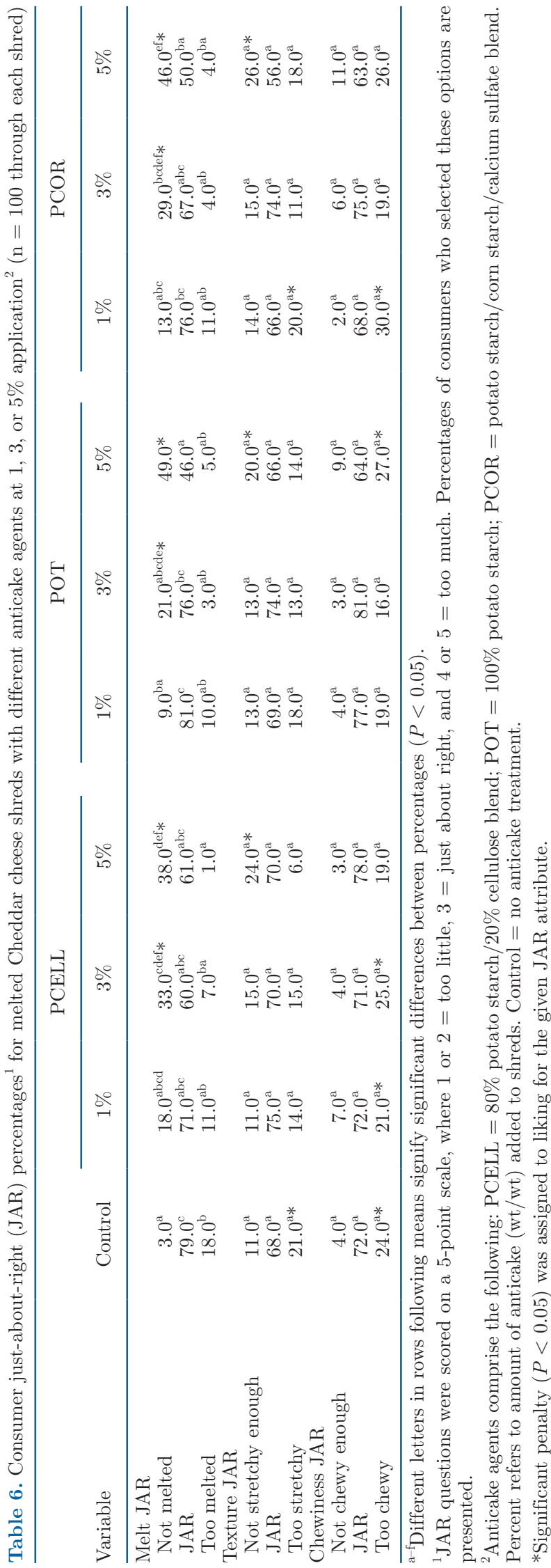


Table 7. Consumer liking means for Cheddar cheese shred home usage test for shreds with 3 different anticake agents at 1 or $5 \%$ application ${ }^{1}$ (wt/wt; $n=49$ through each shred)

\begin{tabular}{|c|c|c|c|c|c|c|}
\hline Item & $\begin{array}{l}\text { Cold } \\
\text { appearance } \\
\text { liking }\end{array}$ & $\begin{array}{l}\text { In-food } \\
\text { appearance } \\
\text { liking }\end{array}$ & $\begin{array}{l}\text { Flavor } \\
\text { liking }\end{array}$ & $\begin{array}{l}\text { Texture } \\
\text { liking }\end{array}$ & $\begin{array}{l}\text { Melt } \\
\text { liking }\end{array}$ & $\begin{array}{l}\text { Stretch } \\
\text { liking }\end{array}$ \\
\hline \multicolumn{7}{|l|}{$\overline{\text { Anticake agent }^{2}}$} \\
\hline $1 \%$ & 7.6 & 7.5 & 7.4 & 7.5 & 7.5 & 7.1 \\
\hline $5 \%$ & 6.6 & 6.9 & 7.1 & 6.9 & 7.0 & 6.7 \\
\hline \multicolumn{7}{|l|}{ POT } \\
\hline \multicolumn{7}{|l|}{ PCOR } \\
\hline $1 \%$ & 7.5 & 7.5 & 7.2 & 7.3 & 7.4 & 6.5 \\
\hline $5 \%$ & 6.4 & 7.3 & 7.4 & 7.4 & 7.4 & 7.1 \\
\hline $\mathrm{LSD}^{3}$ & 0.5 & 0.5 & $\mathrm{NA}^{4}$ & NA & NA & NA \\
\hline \multicolumn{7}{|l|}{$P$-value } \\
\hline Anticake agent & 0.106 & 0.118 & 0.790 & 0.456 & 0.519 & 0.469 \\
\hline Percent application & $<0.001$ & 0.016 & 0.579 & 0.196 & 0.180 & 0.492 \\
\hline
\end{tabular}

as the sample sizes between the 2 tests (100 compared with 49).

When evaluating these results, it is important to remember the practical benefits that anticake agents provide to cheese shreds. Anticake agents provide a functional benefit for manufacture as well as in the consumer supply chain for pre-packaged shreds. Many of the consumer liking scores were significantly different at $95 \%$ confidence; however, the degree of difference for some liking categories was small (0.6 on a 9-point hedonic scale). Consumer results should be viewed with the understanding that statistical differences may not always be practical differences. The largest influence of anticake agents was regarding the cold visual appearance, which is important for initial purchase intent (Speight et al., 2019) and liking before adding the shreds in a food. Industry would benefit from using $100 \%$ potato starch anticake agents for commercially available Cheddar shreds because it had the least influence on cold visual appearance. If Cheddar cheese shreds are to be sold to restaurants or as inclusions, manufacturers can reduce cost by using an anticake agent with reduced potato starch because consumer liking during consumption is less affected by the type of anticake agent. However, adding $>3 \%$ (wt/wt) anticake agent to Cheddar cheese shreds affected several sensory attributes, including cohesiveness (hand and in-mouth), oiliness (visual and to the lips), visual melt- edness, smoothness, and stretch, which may lead to a decrease in consumer satisfaction. Alternatives to anticake agents are conceptually appealing to a clean label, but as the cost of the anticake agent is often less than the cost of the cheese, complete removal of anticake agents from cheese shreds would result in increased costs. Future research should address whether consumers would be willing to pay more for cheese shreds if anticake agents were removed and the cheese remained free flowing.

\section{CONCLUSIONS}

The current study clarified consumer perception of Cheddar cheese shreds with different percent applications and type of anticake agents. Trained panelists documented small effects on melted texture parameters as well as visible cold and hot appearance at applications of $>3 \%$ (wt/wt). These differences were noted by consumers in central location testing and home usage tests by differences in liking scores for shreds with $>3 \%$ (wt/wt) application. Visible appearance was the sensory parameter of Cheddar cheese shreds that was most influenced by anticake agents and percent application. A $100 \%$ potato starch anticake agent applied to Cheddar shreds had the least effect on cold visible liking and the least effect on visible powder and hand cohesiveness descriptive evaluation. However, the $100 \%$ 
potato starch was the most affected anticake agent in the melted shred consumer test and was scored significantly lower than shreds with the other 2 anticake agents in overall appearance and melt liking for the melted CLT at $>3 \%$ application (wt/wt). All 3 consumer tests suggested that application at any percent and type of anticake agent did not greatly influence consumer liking in categories other than visible attributes. The current study aids cheese manufacturers in understanding consumer perception of anticake agents.

\section{ACKNOWLEDGMENTS}

Funding was provided in part by Dairy West (Meridian, ID), the National Dairy Council (Rosemont, IL), Glanbia Nutritionals (Twin Falls, ID), and Schreiber Foods (Green Bay, WI). The authors are grateful to Glanbia Nutritionals (Twin Falls, ID) and Schreiber Foods (Green Bay, WI) for their assistance. The use of trade names does not imply endorsements nor lack of endorsements by those not mentioned. The authors have not stated any conflicts of interest.

\section{REFERENCES}

Akins, M. L. 2002. Effects of starch-based anti-caking agents on the functional properties of shredded mozzarella cheese. MS thesis. Department of Food Science and Technology, Virginia Polytechnic Institute and State University, Blacksburg, VA.

Asato, K. 2003. Lexicon development of appearance and texture descriptors for melted Cheddar cheese using microwave and oven heating. BS thesis. BioResource Research, Oregon State University, Corvallis, OR.

Boutrolle, I., D. Arranz, M. Rogeaux, and J. Delarue. 2005. Comparing central location test and home use test results: Application of a new criterion. Food Qual. Prefer. 16:704-713. https://doi.org/10 .1016/j.foodqual.2005.03.015.

Boutrolle, I., J. Delarue, D. Arranz, M. Rogeaux, and P. Köster. 2007. Central location test vs. home use test: Contrasting results depending on product type. Food Qual. Prefer. 18:490-499. https:// doi.org/10.1016/j.foodqual.2006.06.003.

Brown, J. A., E. A. Foegeding, C. R. Daubert, M. A. Drake, and M. Gumpertz. 2003. Relationships among rheological and sensorial properties of young cheeses. J. Dairy Sci. 86:3054-3067. https:// doi.org/10.3168/jds.S0022-0302(03)73905-8.

Canning, K. 2018. 2018 State of the Industry report: Cheese is a standout on the gridiron. Dairy Foods 119:40-45.

Chappell, R. A., D. A. Grindstaff, and A. R. Woodworth. 2005. Anticaking agents and methods. Allied Starch and Chemical. US Pat. No. 0210694 A1.

Drake, M. A., P. D. Gerard, and G. V. Civille. 1999. Ability of hand evaluation versus mouth evaluation to differentiate texture of cheese. J. Sens. Stud. 14:425-441. https://doi.org/10.1111/j.1745 $-459 X .1999 . t b 00126 . x$.

Drake, M. A., S. C. Mcingvale, P. D. Gerard, K. R. Cadwallader, and G. V. Civille. 2001. Development of a descriptive language for Cheddar cheese. J. Food Sci. 66:1422-1427. https://doi.org/10 $.1111 /$ j.1365-2621.2001.tb15225.x.
Drake, S. L., P. D. Gerard, and M. A. Drake. 2008. Consumer preferences for mild Cheddar cheese flavors. J. Food Sci. 73:S449-S455. https://doi.org/10.1111/j.1750-3841.2008.00960.x.

Elayedath, S., and S. A. Barringer. 2002. Electrostatic powder coating of shredded cheese with antimycotic and anticaking agents. Innov. Food Sci. Emerg. Technol. 3:385-390. https://doi.org/10.1016/ S1466-8564(02)00051-6.

FDA. 2016a. FDA Food Additives Permitted for Direct Addition to Food for Human Consumption. 21CFR172.115. US Food and Drug Administration, Silver Spring, MD.

FDA. 2016b. FDA Generally Recognized as Safe Notice. 21CFR170.255. US Food and Drug Administration, Silver Spring, MD.

Galer, C. D., P. V. Gass, A. S. Handrick, J. A. Hirschey, B. E. LeVine, and C. J. Trinka. 2011. Cheese with improved organoleptic and melting properties. Kraft Foods Global Brands, LLC. US Pat. No. 0244105 A1

Gwartney, E. A., E. A. Foegeding, and D. K. Larick. 2002. The texture of commercial full-fat and reduced-fat cheese. J. Food Sci. 67:812-816. https://doi.org/10.1111/j.1365-2621.2002.tb10682.x.

Lawless, H. T. and H. Heymann. 2010. Descriptive analysis. Pages 227-253 in Sensory Evaluation of Food: Principles and Practices. 2nd ed. Springer, New York, NY.

Meals, S. E., A. E. Schiano, and M. A. Drake. 2020. Drivers of liking for Cheddar cheese shreds. J. Dairy Sci. 103:2167-2185. https:// doi.org/10.3168/jds.2019-16911.

Mintel Group Ltd. 2017. Executive summary - Cheese - US. Accessed Oct. 4, 2017. https://reports.mintel.com/display/794133/.

Mulvany, L. 2016. The Parmesan cheese you sprinkle on your penne could be wood. Accessed Oct. 2020. https://www.bloomberg.com/ news/articles/2016-02-16/the-parmesan-cheese-you-sprinkle-on -your-penne-could-be-wood.

Rogers, N. R., M. A. Drake, C. R. Daubert, D. J. McMahon, T. K. Bletsch, and E. A. Foegeding. 2009. The effect of aging on lowfat, reduced-fat, and full-fat Cheddar cheese texture. J. Dairy Sci. 92:4756-4772. https://doi.org/10.3168/jds.2009-2156.

Smith, G. F., W. Charintranond, P. V. Gass, B. E. LeVine, and A. E. McPherson. 2014. Cheese anticake for enhanced melt. Kraft Foods Global Brands, LLC. US Pat. No. 0272073 A1.

Sosa, M., C. Martinez, F. Marquez, and G. Hough. 2008. Location and scale influence on sensory acceptability measurements among lowincome consumers. J. Sens. Stud. 23:707-719. https://doi.org/10 .1111/j.1745-459X.2008.00181.x.

Speight, K. C., A. N. Schiano, W. S. Harwood, and M. A. Drake. 2019. Consumer insights on prepackaged Cheddar cheese shreds using focus groups, conjoint analysis, and qualitative multivariate analysis. J. Dairy Sci. 102:6971-6986. https://doi.org/10.3168/jds .2018-16209.

Sveinsdóttir, K., E. Martinsdóttir, F. Thórsdóttir, R. Schelvis, A. Kole, and I. Thórsdóttir. 2010. Evaluation of farmed cod products by a trained sensory panel and consumers in different test settings. J. Sens. Stud. 25:280-293. https://doi.org/10.1111/j.1745 $-459 X .2009 .00257 . x$.

USDA Economic Research Service. 2018. Per capita consumption of selected cheese varieties (Annual). Accessed Oct. 8, 2017. https:// www.ers.usda.gov/data-products/dairy-data/.

Zhang, M. T., Y. Jo, K. Lopetcharat, and M. A. Drake. 2020. Comparison of a central location test versus a home usage test for consumer perception of ready-to-mix protein beverages. J. Dairy Sci. 103:3107-3124. https://doi.org/10.3168/jds.2019-17260.

\section{ORCIDS}

M. A. Drake @ https://orcid.org/0000-0002-4744-2493 